\title{
Reflectarray in K and Ka Bands with Independent Beams in Each Polarization
}

\author{
Eduardo Martinez-de-Rioja, Jose A. Encinar
}

\author{
Rafael Florencio, Rafael R. Boix
}

\begin{abstract}
This contribution describes the design of a printed reflectarray antenna to operate at 19.7 and $29.5 \mathrm{GHz}$ with independent beams in $H$ and $V$ polarizations. A dual-frequency dual-polarization reflectarray cell, made of two stacked sets of parallel dipoles for each polarization, is proposed. The dipole lengths are adjusted to produce the required phase-shift at each frequency and each polarization. A $1.6-\mathrm{m}$ reflectarray has been designed to produce two closely spaced beams in $H$ and $V$ polarizations. The simulated radiation patterns show a gain of $48.3 \mathrm{dBi}$ at $19.7 \mathrm{GHz}$ and $50.7 \mathrm{dBi}$ at $29.5 \mathrm{GHz}$, with side-lobe levels close to $-25 \mathrm{~dB}$ and low cross-polar radiation.
\end{abstract}

Keywords - reflectarray, dual polarization, dual frequency, parallel dipoles, Ka-band antennas, satellite antennas.

\section{INTRODUCTION}

The interest on reflectarrays has increased in the last years, due to their low fabrication cost and ease of integration, as well as their capabilities to provide high gain, radiation efficiency and independent beams in each polarization [1]. Reflectarrays have been proposed for multi-frequency operation using a single layer [2] or stacked reflectarray layers for each frequency [3]. A reflectarray that operates at $20 \mathrm{GHz}$ and 30 $\mathrm{GHz}$ in orthogonal linear polarization was reported in [4], by using independent phasing in each polarization. In this case, only one polarization can be used at each frequency. In this paper, the authors present the design of a dual linearly polarized reflectarray to operate in $\mathrm{K}$ and $\mathrm{Ka}$ bands, producing a focused beam at 19.7 and $29.5 \mathrm{GHz}$, with two closely spaced beams in Horizontal (H) and Vertical (V) polarizations.

\section{REFLECTARRAY ELEMENT}

The periodic element comprises two orthogonal sets of five parallel dipoles printed on a dielectric layer, and two additional sets of three parallel dipoles, which are stacked above the first sets and are printed on the top of a second dielectric sheet (see Fig.1). The lateral dipoles are symmetric with respect to the central one, in order to keep low levels of cross-polarization. The period is chosen as $\mathrm{P}_{\mathrm{X}}=\mathrm{P}_{\mathrm{Y}}=6.5 \mathrm{~mm}$ to avoid the appearance of grating lobes at the higher frequency $(29.5 \mathrm{GHz})$. The electrical properties of both layers are $\varepsilon_{\mathrm{rP}}=\varepsilon_{\mathrm{rQ}}=2.17, \tan (\delta)_{\mathrm{P}}=\tan (\delta)_{\mathrm{Q}}=0.002$, and their thickness is $h_{\mathrm{P}}=1.5 \mathrm{~mm}, h_{\mathrm{Q}}=1 \mathrm{~mm}$. The dipole width is $w=0.25 \mathrm{~mm}$, separations between laterally coupled dipoles are $\mathrm{S}_{\mathrm{XP}}=\mathrm{S}_{\mathrm{YP}}=$
$0.5 \mathrm{~mm}, \mathrm{~S}_{\mathrm{XQ}}=\mathrm{S}_{\mathrm{YQ}}=1 \mathrm{~mm}$, and relative sizes of lateral dipoles are $l_{\mathrm{P} 1}=0.65 \cdot l_{\mathrm{P} 3}, l_{\mathrm{P} 2}=0.8 \cdot l_{\mathrm{P} 3}, l_{\mathrm{P} 4}=0.65 \cdot l_{\mathrm{P} 6}, l_{\mathrm{P} 5}=0.8 \cdot l_{\mathrm{P} 6}$ (where $l_{\mathrm{P} 3}$ and $l_{\mathrm{P} 6}$ correspond to the lengths of the central dipoles), $l_{\mathrm{Q} 1}=0.8 \cdot l_{\mathrm{Q} 2}, \quad l_{\mathrm{Q} 3}=0.8 \cdot l_{\mathrm{Q} 4}$ (where $l_{\mathrm{Q} 2}$ and $l_{\mathrm{Q} 4}$ correspond to the central dipoles lengths). These parameters have been chosen to provide a smooth variation in phase response in a range greater than $360^{\circ}$ at $19.7 \mathrm{GHz}$ and 29.5 $\mathrm{GHz}$. Lower layer dipoles will be longer than those on the top layer, so that upper dipoles will not disturb the reflectarray phase response at $19.7 \mathrm{GHz}$, while lower dipoles will behave as a ground plane at $29.5 \mathrm{GHz}$ for higher layer elements. A practically independent phase control based on upper and lower dipoles can be observed in Fig. 2, which shows the phase variation of the reflection coefficient at 19.7 and 29.5 $\mathrm{GHz}$ for H-polarization under normal incidence (note that the same response will be obtained for $\mathrm{V}$-polarization). Therefore, the dipole lengths can be obtained separately for each band: first, the dipoles on the bottom layer are adjusted to produce the required phase-shift at $19.7 \mathrm{GHz}$, and then those on the top layer are adjusted to provide the required phase at $29.5 \mathrm{GHz}$. This factor will allow for performing an easier and computationally faster design process.

\section{ANTENNA DESIGN}

A circular reflectarray, consisting of 49,080 elements arranged in a $250 \times 250$ grid (162.5-cm diameter), has been designed to generate a focused beam in the direction $\theta_{\mathrm{b}}=10^{\circ}$, $\varphi_{\mathrm{b}}=0^{\circ}$ for H-polarization and a closely spaced beam at $\theta_{\mathrm{b}}=10.5^{\circ}, \varphi_{\mathrm{b}}=0^{\circ}$ for $\mathrm{V}$-polarization at both frequency bands, which are transmit and receive frequencies for multi-spot satellite antennas in Ka-band. The antenna is illuminated by a feed-horn (see Fig. 3), whose phase center is placed at coordinates $(-30,0,100) \mathrm{mm}$ with respect to the reflectarray center. The field radiated by the horn is modelled using a $\cos ^{\mathrm{q}}(\theta)$ function, with $\mathrm{q}=6$ for $20 \mathrm{GHz}$ band and $\mathrm{q}=8$ for 30 $\mathrm{GHz}$ band. The edge illumination level is close to $-16 \mathrm{~dB}$ at 19.7 GHz and $-18 \mathrm{~dB}$ at $29.5 \mathrm{GHz}$. An iterative zero-finding procedure is employed to adjust the dipole lengths, which calls a home-made software analysis routine, based on the Method of Moments in the Spectral Domain (MoM-SD) and the local periodicity approach [5]. This routine takes into account the real angle of incidence $\theta_{\mathrm{i}}$ on each reflectarray element to calculate the amplitude and phase of the reflection coefficient, considering the element in a periodic environment. 


\section{Simulation Results}

The simulated radiation patterns in gain, shown in Fig. 4, have been obtained from the tangential reflected field at each reflectarray element, using the previously mentioned MoM-SD software. A gain of $48.3 \mathrm{dBi}$ is attained at $19.7 \mathrm{GHz}$, and 50.7 $\mathrm{dBi}$ gain is reached at $29.5 \mathrm{GHz}$, with low levels for crosspolar components (around $30 \mathrm{~dB}$ below the co-polar maximum). The beam width at $-3 \mathrm{~dB}$ is lower than $0.7^{\circ}$, and side-lobe level is close to $-30 \mathrm{~dB}$ and $-25 \mathrm{~dB}$ at $19.7 \mathrm{GHz}$ and $29.5 \mathrm{GHz}$, respectively. The antenna radiation efficiency can be estimated as $66 \%$ in $\mathrm{K}$ band and $48 \%$ in Ka band, which are typical values for conventional reflectors (the simulations account for illumination, spillover and dielectric losses).

\section{CONCLUSIONS}

The results show the potential of reflectarray antennas for working at different bands, with independent beam-shaping in each polarization and band. The reflectarray element based on two stacked layers with sets of parallel dipoles enables simultaneous phase adjustment at both design frequencies. The simulated radiation patterns are very promising for multi-spot antennas in satellites that operate in $\mathrm{K}$ and $\mathrm{Ka}$ bands.

\section{ACKNOWLEDGMENT}

This work has been supported by the Spanish Ministry of Economy and Competitiveness under the project TEC201343345-P.

\section{REFERENCES}

[1] J. Huang and J. A. Encinar, "Reflectarray Antennas", IEEE Press/Wiley, Piscataway, New Jersey, 2008.

[2] H. Hasani, C. Peixeiro, A. Skrivervik, J. Perruisseau-Carrier, "SingleLayer Quad-Band Printed Reflectarray Antenna with Dual Linear Polarization," IEEE Trans. on Antennas and Propag., vol.63, no.12, pp. 5522-5528, Dec. 2015

[3] M. R. Chaharmir, J. Shaker, H. Legay, "Dual-band Ka/X reflectarray with broadband loop elements", Microwaves, Antennas \& Propagation, IET, vol. 4, no. 2, pp. 225-231, Feb. 2010.

[4] J. A. Encinar, M. Barba, "Design manufacture and test of Ka-band reflectarray antenna for transmitting and receiving in orthogonal polarization", in 14th International Symposium on ANTEM-AMEREM, 2010, Ottawa, Canada, 5-8 July 2010.

[5] R. Florencio, J. A. Encinar, R. R. Boix, V. Losada, G. Toso, "Reflectarray Antennas for Dual Polarization and Broadband Telecom Satellite Applications," IEEE Trans. on Antennas and Propag., vol. 63, pp. 1234-1246, April 2015.

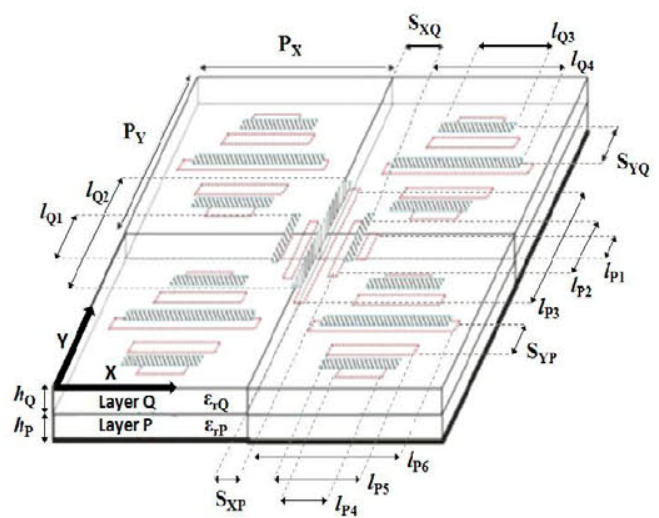

Fig. 1. View of reflectarray periodic structure, which includes four cells for $\mathrm{H}$-polarization and one cell for $\mathrm{V}$ polarization.

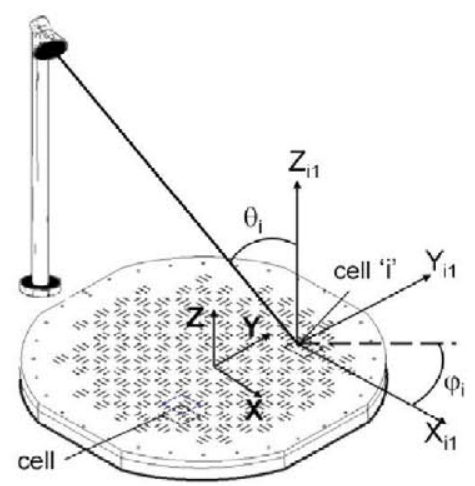

Fig. 3. Reflectarray antenna showing the reference coordinate systems.

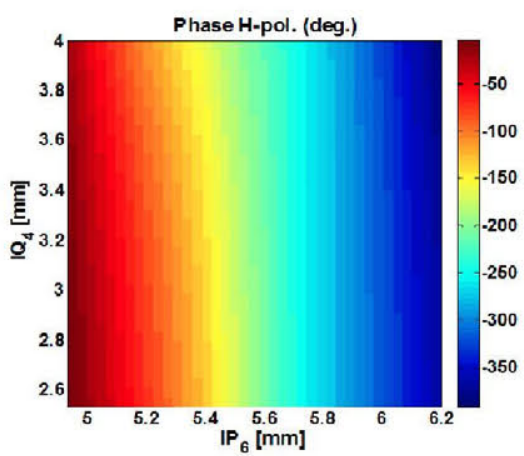

(a)

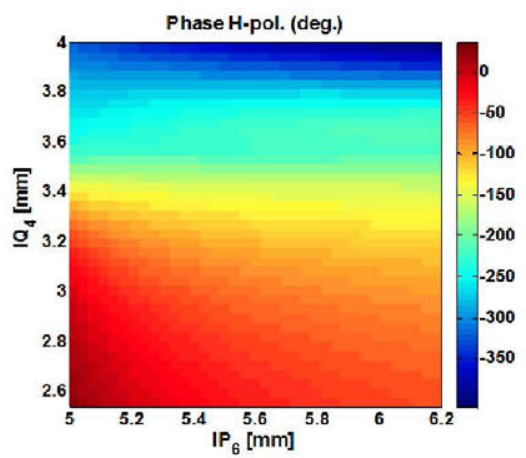

(b)
Fig. 2. Phase variation of the co-polar reflection coefficient for H-polarization and normal incidence, regarding the lengths of the dipoles in X-direction: (a) at $19.7 \mathrm{GHz}$, (b) at $29.5 \mathrm{GHz}$.

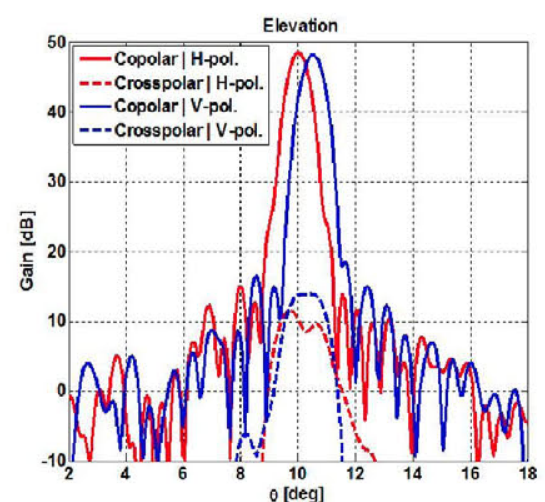

(a)

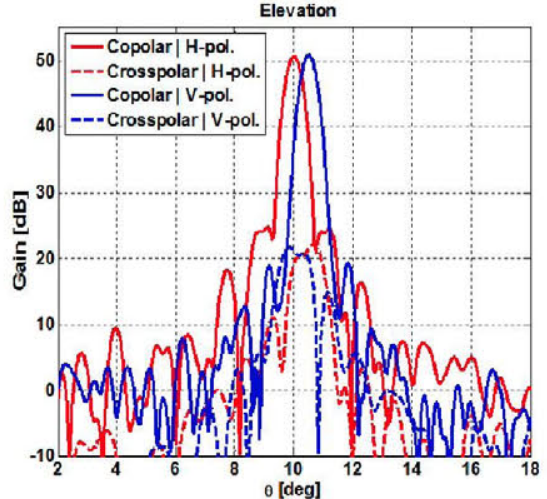

(b)
Fig. 4. Simulated radiation patterns in gain for co-polar and cross-polar components: (a) XZ-plane at $19.7 \mathrm{GHz}$, (b) XZ-plane at $29.5 \mathrm{GHz}$. 